\title{
Bacurd2 is a novel interacting partner to Rnd2 which controls radial migration within the developing mammalian cerebral cortex
}

Ivan Enghian Gladwyn- $\mathrm{Ng}^{1 \dagger}$, Shan Shan $\mathrm{Li}^{1 \dagger}$, Zhengdong $\mathrm{Qu}^{1 \dagger}$, John Michael Davis ${ }^{1}$, Linh $\mathrm{Ngo}^{1,3}$, Matilda Haas ${ }^{1}$, Jeffrey Singer ${ }^{2}$ and Julian Ik-Tsen Heng ${ }^{1,3,4,5^{*}}$

\begin{abstract}
Background: During fetal brain development in mammals, newborn neurons undergo cell migration to reach their appropriate positions and form functional circuits. We previously reported that the atypical RhoA GTPase Rnd2 promotes the radial migration of mouse cerebral cortical neurons (Nature 455(7209):114-8, 2008; Neuron 69(6):1069-84, 2011), but its downstream signalling pathway is not well understood.

Results: We have identified BTB-domain containing adaptor for Cul3-mediated RhoA degradation 2 (Bacurd2) as a novel interacting partner to Rnd2, which promotes radial migration within the developing cerebral cortex. We find that Bacurd2 binds Rnd2 at its C-terminus, and this interaction is critical to its cell migration function. We show that forced expression or knockdown of Bacurd2 impairs neuronal migration within the embryonic cortex and alters the morphology of immature neurons. Our in vivo cellular analysis reveals that Bacurd2 influences the multipolarto-bipolar transition of radially migrating neurons in a cell autonomous fashion. When we addressed the potential signalling relationship between Bacurd2 and Rnd2 using a Bacurd2-Rnd2 chimeric construct, our results suggest that Bacurd2 and Rnd2 could interact to promote radial migration within the embryonic cortex.
\end{abstract}

Conclusions: Our studies demonstrate that Bacurd2 is a novel player in neuronal development and influences radial migration within the embryonic cerebral cortex.

Keywords: Neuronal migration, Cerebral cortex, Rho GTPase, Bacurd2, Tnfaip1, Rnd2

\section{Background}

During mammalian brain development, newborn neurons undergo a well-defined migratory journey in order to arrive at their final location within the developing nervous system and form functional connections with other neural cells [1-3]. Following their birth within the germinal zone of the ventricular neuroepithelium (known as the ventricular zone (VZ)), they migrate through a transitional intermediate zone (IZ) before arriving at their appropriate positions within the cortical plate $(\mathrm{CP})$ and undergo terminal differentiation. Failure in the proper positioning of neurons during brain development can result

\footnotetext{
* Correspondence: julian.heng@perkins.uwa.edu.au

${ }^{\dagger}$ Equal contributors

'EMBL Australia, The Australian Regenerative Medicine Institute, Monash University, Clayton, Victoria 3800, Australia

${ }^{3}$ The Harry Perkins Institute of Medical Research, Perth, Australia

Full list of author information is available at the end of the article
}

in the formation of abnormal neural circuits, leading to intellectual impairment and epilepsy in humans $[4,5]$.

While the molecular mechanisms which govern cell migration during brain development are not fully understood, recent work has revealed that neuronal migration is intrinsically regulated by the activity of DNA binding transcription factors on a RhoA-like GTPase gene known as Rnd2 [6,7]. It was discovered that members of the basic helix-loop-helix (bHLH) family of transcriptional activators (such as Neurog2, NeuroD1 and NeuroD2) stimulate Rnd2 expression to promote the migration of newborn excitatory neurons of the cerebral cortex $[6,8]$. Furthermore, transcriptional repressors such as COUP-TFI and RP58 negatively regulate $R n d 2$ expression in the course of their radial migration and control their multipolar-to-bipolar conversion within the IZ as they enter the $\mathrm{CP}$ to complete their migration [9-11]. Together, these multiple regulatory 
pathways control appropriate levels of Rnd2 gene dosage in neurons to shape their development during cortical neurogenesis.

Despite a deep understanding of the regulation of Rnd2 expression for the positioning of neurons within the nascent cortex, the intracellular signalling pathways through which Rnd2 controls cell migration remain less well understood. Nevertheless, Rnd2 and its related family member Rnd3 are both known to control radial migration and neurite outgrowth through their actions on the actin cytoskeleton $[6,7,12]$. However, while recent studies demonstrate that both Rnd proteins commonly suppress RhoA signalling and modulate the filamentous-actin (F-actin) cytoskeleton within cortical neurons as they differentiate within the embryonic cortex [7], the underlying signalling mechanisms for Rnd2 and Rnd3 are known to be different. Notably, Rnd3 mediates actin depolymerisation and promotes cell migration within the embryonic cortex through its downstream effector molecule p190RhoGAP, while Rnd2 does not signal through this pathway [7]. In addition, Rnd proteins are known to interact with different protein partners in order to elicit their effects on fibroblast cell shape and motility (reviewed in $[13,14]$ ), thus the challenge remains to better understand the complexity of the downstream signalling pathways through which Rnds function in neural cells as well.

In this study, we wanted to clarify the signalling pathway through which Rnd2 mediates cell migration during neuronal development in mice. We have identified a member of the BTB-domain containing adaptor for Cul3mediated RhoA degradation (Bacurd2) as a novel binding partner to Rnd2 within the mouse embryonic cerebral cortex. We report that knockdown or forced expression of Bacurd2 disrupts radial cell migration in vivo and that Bacurd2 promotes the multipolar-to-bipolar transition of neurons as they transit from the intermediate zone into the cortical plate. In our exploration of the functions for Bacurd2 and Rnd2, we find both to be crucial to the migration of newborn neurons within the embryonic cerebral cortex.

\section{Results}

Bacurd2 interacts with Rnd2 and mediates cell migration within the embryonic cerebral cortex

To identify binding partners to Rnd2, we performed a yeast two-hybrid screen of an embryonic mouse (E15.5) cortex library [15] using an Rnd2 bait construct lacking the C-terminal membrane-binding (CAAX) motif. A survey of $2 \times 10^{7}$ independent clones resulted in the isolation of multiple interacting prey clones encoding polypeptides corresponding to full-length Bacurd2, as well as a smaller fragment comprising the C-terminal aa242-316 fragment. Following prey plasmid recovery, complementation tests confirm specificity of interaction between Bacurd2 preys and the Rnd2 bait, but not pLaminC or with p53 (Additional file 1: Figure S1). To confirm protein-protein interaction between Bacurd2 and Rnd2, we performed immunoprecipitation experiments with epitope-tagged constructs and found that FLAG-tagged Rnd2 binds to EGFP-Bacurd2 fusion protein, but not to EGFP alone (Figure 1A). We also performed immunoprecipitation experiments with mouse embryonic (E14.5) brain lysate using a Bacurd2 antibody (Additional file 2: Figure S2A) to confirm their interaction in vivo (Figure 1B). Bacurd2 and Rnd2 are detected throughout the course of brain development (Additional file 2: Figure S2C). Immunostaining of embryonic E14.5 cerebral cortex tissue revealed Bacurd2 signal in the VZ, sVZ and IZ, while parallel experiments performed with pre-immune serum did not elicit a signal (Additional file 2: Figure S2D-E).

Next, we performed a series of in utero electroporation experiments on E14.5 mouse embryonic cortex to determine whether perturbations to Bacurd 2 might disrupt cortical development. To do this, we forced expressed Bacurd2 by delivering a bicistronic expression construct encoding Bacurd2 and GFP into embryonic cortical cells and examined the distribution of GFP-labelled cells 3 days later at E17.5. In a reciprocal approach, we suppressed Bacurd2 expression in cells using targeting siRNAs together with an empty (GFP only) vector (Figure 2A). In each condition, the amounts of siRNA (control or targeting) and expression vector (GFP only, or GFP + Bacurd2 bicistronic vector) were normalised to enable comparisons across conditions. In Figure 2B, we show that while a significant proportion of GFP-labelled cells had migrated into the CP of control-treated brains, forced expression of Bacurd2 or knockdown with siRNAs disrupted their migration within the embryonic cortex, observed as an accumulation of cells within the IZ and a concomitant decrease in cells located within the CP (Figure 2C). Within the CP, a significant proportion of Bacurd2overexpressing cells and Bacurd2 siRNA-treated cells failed to reach the upper cortical plate, suggesting that changes to Bacurd2 levels disrupt their 'intracortical' positioning (Figure 2D). To account for the possibility that disruptions to Bacurd2 might influence cortical neurogenesis, we performed quantification studies and found no significant differences in the proportions of GFP+/Tuj1+ cells or their distribution within the subcompartments of the embryonic E17.5 cortex (Additional file 3: Figure S3).

To confirm the specificity of the siRNA-mediated migration defect, we performed rescue experiments whereby cells were co-treated with an expression construct encoding human BACURD2 which was refractory to RNAi (Figure 3). Our results show that the defective migration of siRNA-treated cells could be significantly restored to levels resembling control condition when $0.4 \mu \mathrm{g} / \mu \mathrm{l}$ of 
A

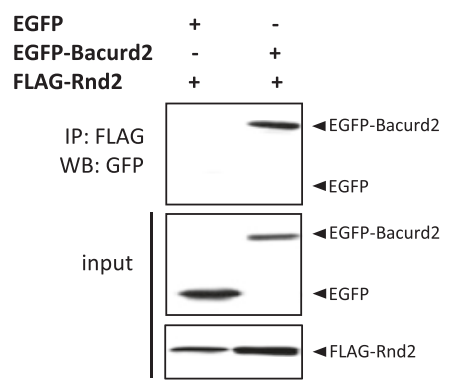

B

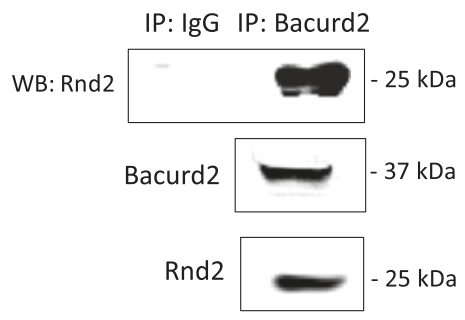

Figure 1 Bacurd2 interacts with Rnd2 in vitro and in vivo. (A) Immunoprecipitation assays with transiently transfected HEK293T cells show that FLAG-Rnd2 interacts with EGFP-Bacurd2, but not EGFP. (B) Lysates of E14.5 mouse brain homogenates were immunoprecipitated with a Bacurd2 antibody and probed by immunoblotting for Rnd2 to confirm their interaction in vivo. As a control, mouse-anti-lgG did not immunoprecipitate Rnd2. Input lanes confirm protein expression in both experiments. Further details of antibodies used to detect Bacurd2 and Rnd2 are provided in Additional file 2: Figure $\mathrm{S} 2$.

BACURD2 construct was co-delivered with Bacurd2 siRNA (Figure 3C). Interestingly, while co-treatment with either concentrations of BACURD2 enhanced migration into the $\mathrm{CP}$ (Additional file 4: Figure S4), we show in Figure 3C,D that the migration profile of siRNAtreated cells was corrected to levels resembling control when co-treated with $0.4 \mu \mathrm{g} / \mu \mathrm{l}$ of BACURD2 (Figure 3C), while co-treatment with a higher concentration $(1 \mu \mathrm{g} / \mu \mathrm{l})$ of BACURD2 construct disrupted intracortical positioning (Figure 3D). Thus, Bacurd2 cell autonomously controls radial migration, with concentration-sensitive effects.

In the course of their radial migration, embryonic cortical cells adopt different modes of migration from the germinal VZ, through to the IZ and the CP $[16,17]$. Hence, we analysed the morphology of GFP-labelled neurons to describe the cellular basis for the defective migration of cells as a result of perturbations to Bacurd2. Within the IZ, we found that forced expression of Bacurd 2 resulted in a significant increase in the proportion of round-shaped cells which have very short processes (or no detectable processes at all), together with a corresponding decrease in multipolar-shaped neurons; while the proportion of uni/bipolar-shaped neurons was not significantly different (Figure 4). On the other hand, knockdown of Bacurd2 resulted in a significant increase in the proportion of multipolar-shaped neurons and a concomitant decrease in uni/bipolar neurons, while the proportion of round-shaped neurons was not significantly different. Within the $\mathrm{CP}$, we found that forced expression as well as knockdown of Bacurd2 resulted in an increase in the proportion of round-shaped cells, together with a decrease in the proportions of uni/bipolar-shaped cells. These documented changes in cell morphology upon siRNA-mediated knockdown were corrected by codelivery of $0.4 \mu \mathrm{g} / \mu \mathrm{l}$ BACURD2 construct (Additional file 5: Figure S5). Together, these results demonstrate that disruptions to Bacurd2 alter the morphologies of embryonic neurons, and this effect could underlie their defective migration within the embryonic E17.5 cortex.

In the following experiments, we wanted to define the interaction domains on Bacurd2 which govern its binding to Rnd2. We cloned truncation mutants of Bacurd2 based on the minimal interaction regions identified in our yeast two-hybrid assay (Additional file 1: Figure S1) and assessed their interaction in co-immunoprecipitation assays using epitope-tagged proteins in heterologous cells (Figure 5). Our results show that while a C-terminal truncation mutant Bacurd2( $\Delta 221-316)$ fails to immunoprecipitate Rnd2, an N-terminal mutant Bacurd2( $\Delta 1-109)$ still interacts with Rnd2 (Figure 5B, lanes 3 to 4). Recently, Bacurd2 was demonstrated to interact with the E3 ubiquitin ligase $\mathrm{Cul} 3$ at its $\mathrm{N}$-terminus and signal together to promote fibroblast cell migration in vitro [18]. Given that Bacurd2, Rnd2 and Cul3 proteins are all present during mouse brain development (Additional file 2: Figure S2C), we wanted to confirm their protein-protein interaction. As shown, our co-immunoprecipitation experiments reveal that while Cul3 interacts with full-length Bacurd2, as well as a $\mathrm{C}$-terminal truncation mutant, the $\mathrm{N}$-terminal mutant Bacurd2( $\Delta 1-109)$ fails to immunoprecipitate Cul3 (Figure $5 \mathrm{C}$ ). In addition, we engineered missense mutations I71A/L72A/I73A to Bacurd2 (named as Bacurd2(3A), the location of these amino acids are indicated in bold text on Figure 5A) which are reported to disrupt its BTB domain [18], and we found that this variant did not interact with Cul3 (Figure 5D). Therefore, these studies demonstrate that Bacurd2 interacts with Rnd2 as well as $\mathrm{Cul} 3$ via the $\mathrm{C}$ - and $\mathrm{N}$-termini, respectively (summarised in Figure 5E).

Next, we investigated how the Bacurd2 polypeptide influences neuronal migration by performing in utero electroporation assays. Specifically, we asked if forced expression of each of the mutated variants of Bacurd2 (that is Bacurd2( $\Delta 1-109)$, Bacurd2(3A) and 

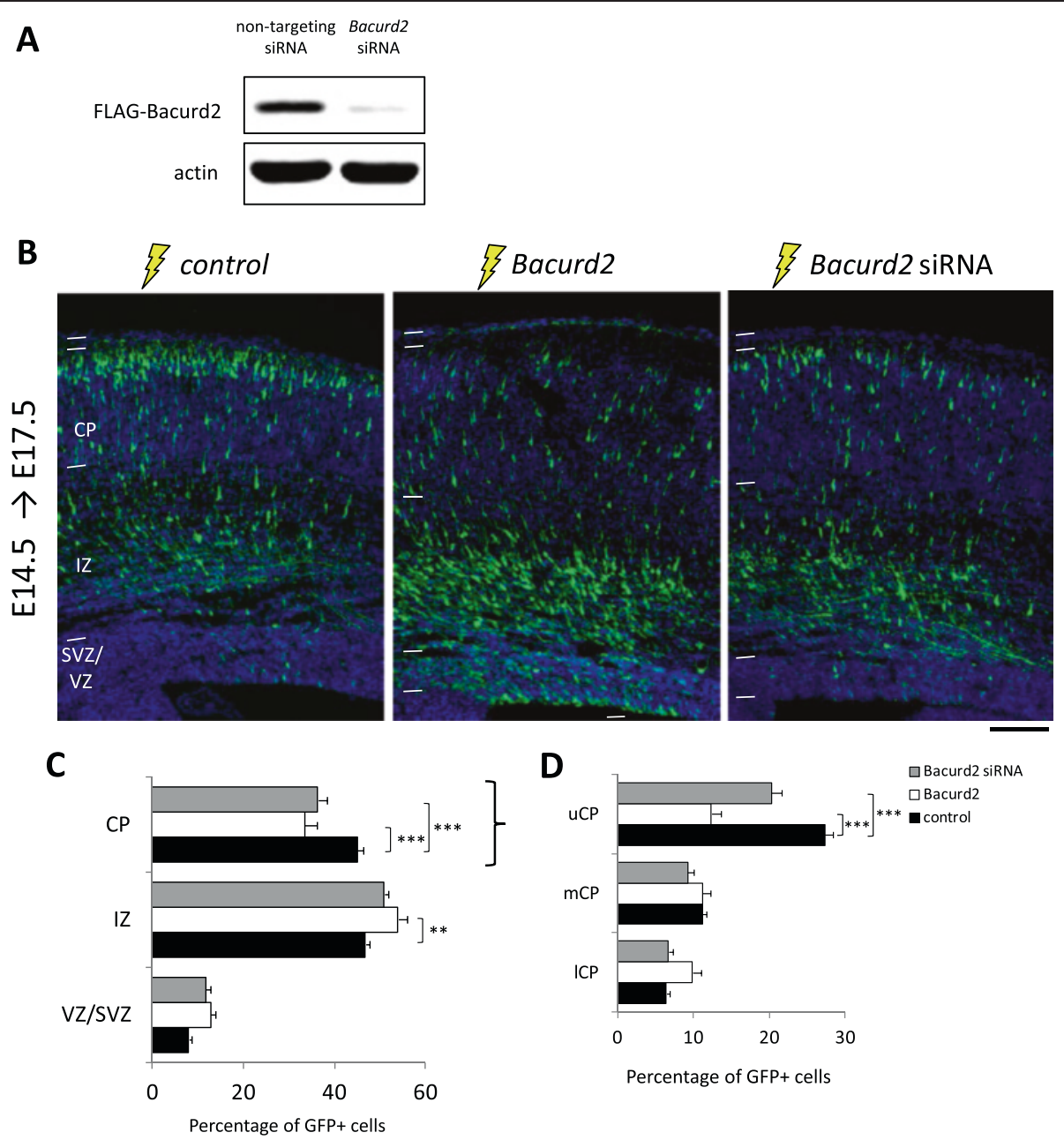

Figure 2 Bacurd2 influences cell migration within the embryonic mouse cerebral cortex. (A) Western blotting with HEK293T cell lysates confirms that FLAG-Bacurd2 expression is suppressed by targeting siRNAs, but not by control (non-targeting) siRNAs. Actin was used as loading control. (B) In utero electroporation was performed on embryonic mouse E14.5 embryos and analysed 3 days later at E17.5. Cortical cells were electroporated with control vector (GFP only), a bicistronic GFP expression construct which also encodes Bacurd2, or Bacurd2 siRNA co-electroporated with GFP vector. (C) Quantification reveals that forced expression of Bacurd2, or treatment with Bacurd2 siRNAs, alters the distribution of cells within the embryonic cortex $\left(N>4,500\right.$ cells from four to six brains per condition; $F_{4,72}=14.97 ; P<0.0001$; two-way ANOVA followed by Bonferroni's post hoc test; ***P $<0.0001$. (D) Quantification of GFP+ cells within the CP (divided into the lower, medial and upper CP) reveals that forced expression of Bacurd2 or knockdown of Bacurd2 disrupts the intracortical distribution of GFP+ cells compared with control ( $N>1,500$ cells from four to six brains per condition; $F_{4,72}=27.89 ; P<0.0001$; two-way ANOVA followed by Bonferroni's post hoc test). UCP, $\mathrm{mCP}$ and ICP indicate upper, medial and lower cortical plates, respectively. Scale bar represents $100 \mu \mathrm{m}$.

Bacurd2( $\Delta 221-316))$ might affect the migration of E14.5 embryonic cortical cells within the E17.5 cortex in a similar manner to wildtype Bacurd2. As shown in Figure 6, we found that while forced expression of full-length Bacurd2 disrupted the migration of embryonic cortical cells into the $\mathrm{CP}$, forced expression of the $\mathrm{N}$-terminal truncation mutants Bacurd2( $11-109)$ or Bacurd2(3A) (both of which fail to interact with Cul3) did not significantly disrupt the migration profile of treated cells when compared with control. Similarly, forced expression of the Rnd2-binding defective mutant Bacurd2( $\Delta 221-316)$ mutant did not significantly disrupt the migration profile of GFP-labelled cells. Therefore, overexpression of all three mutants did not disturb migration and this suggests that an intact, fulllength Bacurd2 polypeptide is important for its cell migration functions within the embryonic cortex.

\section{A Bacurd2:Rnd2 chimeric construct influences radial migration within the embryonic cortex}

Based on our analysis of Bacurd2 and its mutants in migration (Figure 6), we reasoned that the Bacurd2 polypeptide must coordinate cell migration through its protein-protein interactions at its $\mathrm{N}$ - and $\mathrm{C}$-termini. To explore the possibility that Bacurd2 might signal cell migration in concert with Rnd2, we designed a polypeptide expression construct comprising a fusion between 
A
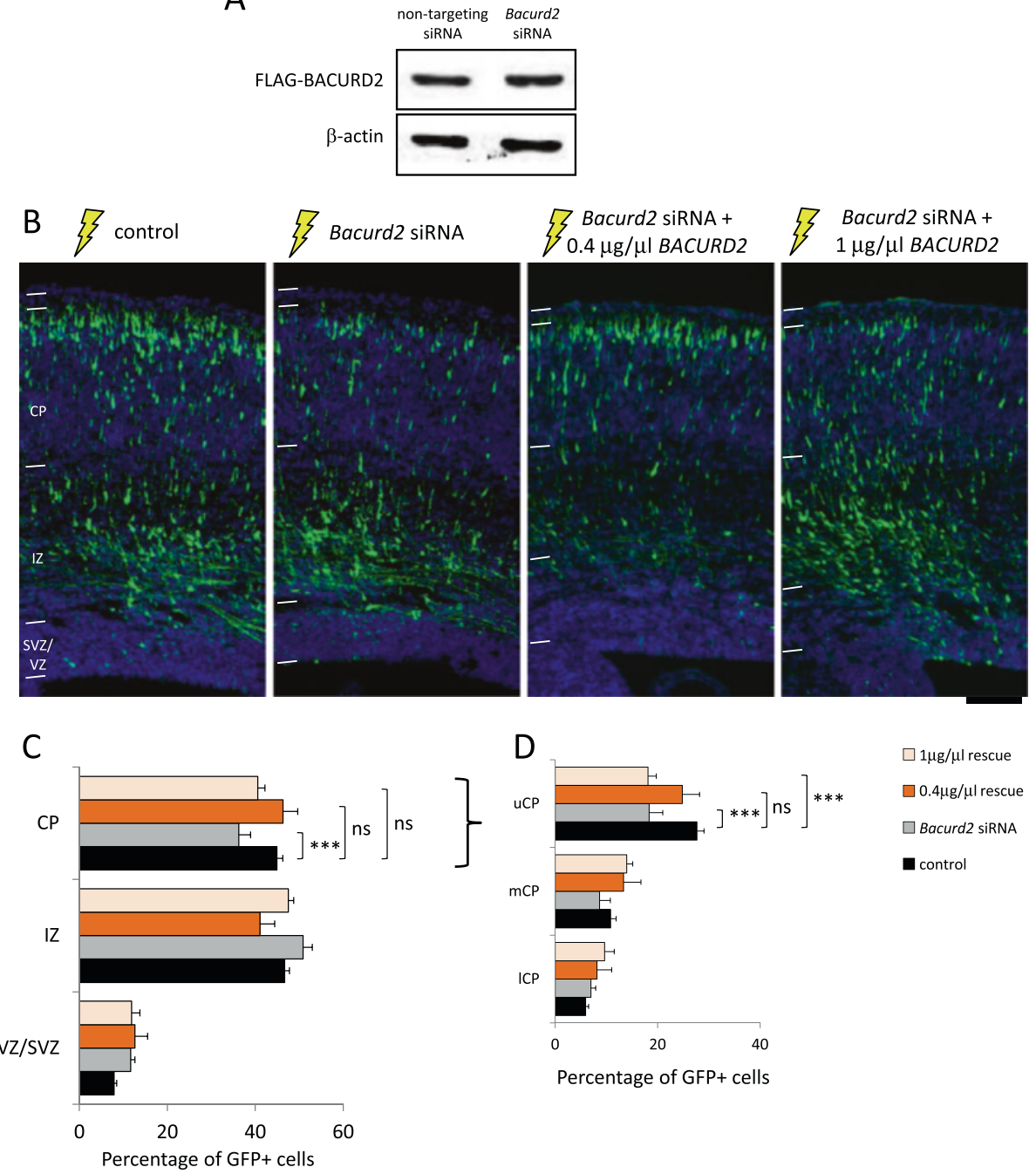

Figure 3 The defective migration of Bacurd2 siRNA-treated cells is augmented by co-delivery of human BACURD2. (A) Western blotting of lysates from P19 embryocarcinoma cells transiently transfected with control siRNA or Bacurd2 siRNAs, together with an expression construct encoding human BACURD2 as an epitopte-tagged (FLAG) protein. FLAG-BACURD2 protein expression is refractory to Bacurd2 siRNA-mediated knockdown. (B) In utero electroporation studies with E14 mouse brains electroporated with GFP vector and control siRNA ('control'), GFP vector and Bacurd2 siRNA, and Bacurd2 siRNA with the indicated concentrations of BACURD2 expression construct are indicated. (C) Quantitation reveals that while Bacurd2 siRNA treatment impairs radial migration, co-delivery of $0.4 \mu \mathrm{g} / \mu \mathrm{l}$ BACURD2 construct restores their migration to control levels, while co-delivery of $1.0 \mathrm{\mu g} / \mu \mathrm{l}$ BACURD2 construct only partially restores their migration within the embryonic cortex ( $N>1,450$ cells counted per condition; $F_{6.45}=15 ; P<0.0001$; two-way ANOVA followed by Bonferroni's post hoc test). (D) An analysis of their intracortical distribution reveals that the defective migration of Bacurd2 siRNA-treated cells is restored with co-delivery of $0.4 \mu \mathrm{g} / \mu \mathrm{l}$ of BACURD2 construct ( $N>500$ cells per condition; $F_{6,42}=15 ; P<0.0001$; two-way ANOVA followed by Bonferroni's post hoc test). Graph plots mean \pm SEM. Scale bar represents $100 \mu \mathrm{m}$.

the N-terminal Bacurd2(aa1-220) sequence together with the C-terminal sequence of Rnd2(aa181-227) (Figure 7A). It was recently discovered that the C-terminal (aa181-227) region of Rnd2 is important for signalling cell migration in vivo [7], and so we cloned this region of Rnd2 in place of Bacurd2(aa221-316) to generate a chimeric molecule. When we introduced this construct into E14.5 born cortical cells, we found that forced expression of the Bacurd2:Rnd2 disrupts radial migration in a manner which was distinct to Rnd2 or Bacurd2 overexpression alone (Figure 7B). Notably, we found that forced expression of Bacurd2 led to a significant accumulation of cells in the IZ and a failure of cells to reach the CP, while forced expression of Rnd2 resulted in a significant accumulation of cells in the VZ but not the IZ. In contrast, forced expression of Bacurd2:Rnd2 led to a significant accumulation of cells in the VZ and IZ. Consistent with these distinct effects on cell migration, we found that each different treatment altered the morphology of IZ and CP cells in different ways (Additional file 6: Figure S6). 


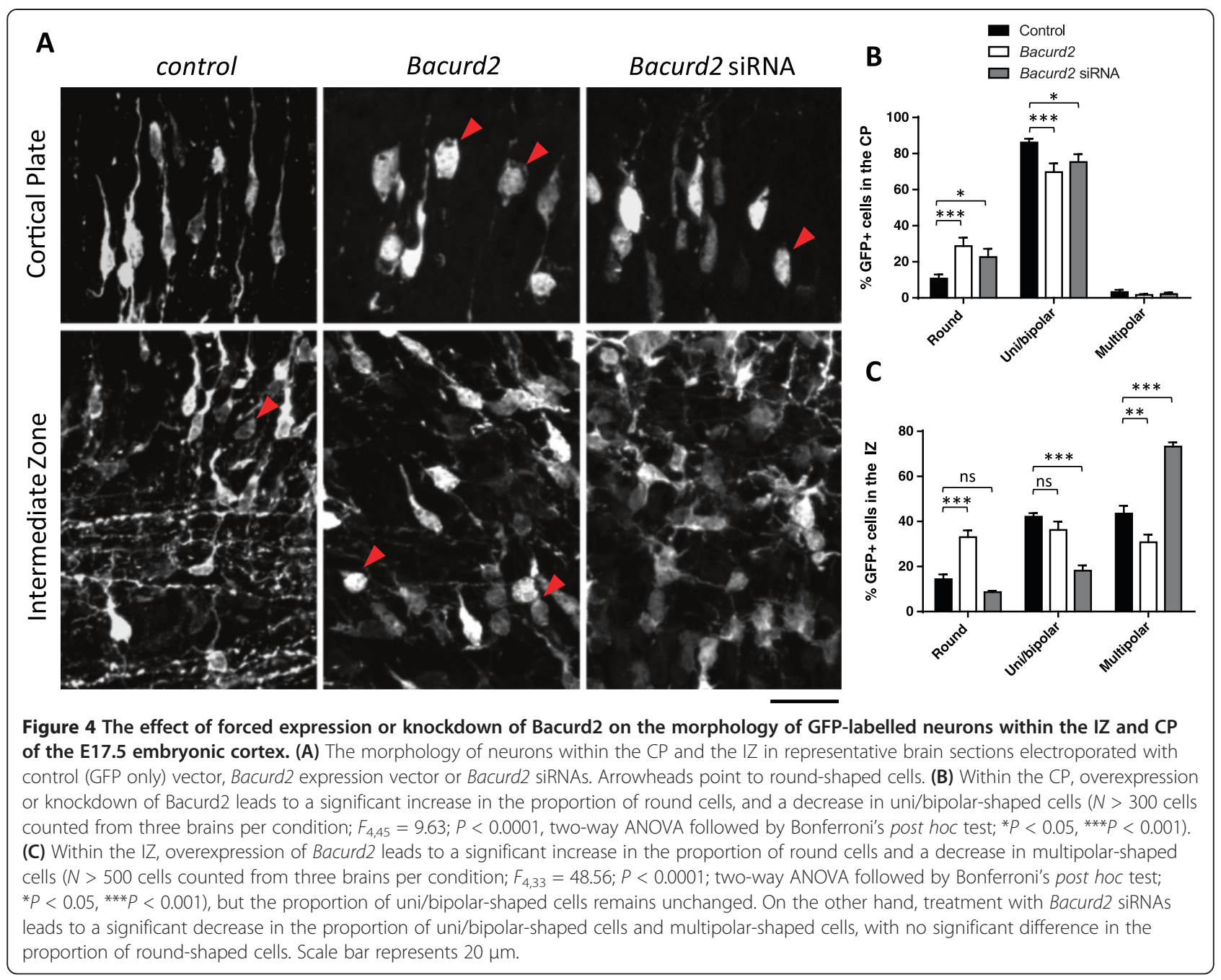

It was reported that suppression of Rnd2 by RNAi significantly disrupted cell migration within the embryonic E17.5 cortex, including their multipolar-to-bipolar transition from the IZ to the CP $[6,7,10]$. Hence, we wanted to determine if the migration defect of Rnd2-deficient cells could be restored by modulating Bacurd2 signalling. We began with control experiments to confirm that the defective migration of Rnd2 shRNA-treated cells could be corrected by co-delivering an expression construct encoding Rnd 2 which is not targeted by the shRNA vector (Figure 8A,B) [6,7]. Next, we asked whether forced expression of full-length Bacurd2 could compensate for the defective migration of Rnd2 shRNA-treated cells, but we did not observe a restoration of cell migration in our assay ( $n=6$ brains per condition, data not shown). In contrast, co-delivery of Bacurd2:Rnd2 significantly improved the migration of Rnd2 shRNA-treated cells (Figure 8A), with cells reaching the cortical plate at levels not significantly different to control treatment (Figure 8B; 39.37\% \pm $2.57 \%$ of cells within the $\mathrm{CP}$ of control samples versus
$33.45 \% \pm 2.4 \%$ of $R n d 2$ shRNA + Bacurd2:Rnd2 treated cortices; $F_{8,39}=17.36 ; P<0.0001$; two-way ANOVA; post hoc $t$-test $P>0.05$ ). In addition to this result, we were also interested to determine whether I71A/L72A/I73A substitution mutations to the BTB domain of Bacurd2 which disrupt its binding to $\mathrm{Cul} 3$ were relevant to its cell migration functions. Thus, we performed parallel rescue experiments to co-deliver Bacurd2(3A):Rnd2 (which is defective in Cul3 binding; see Additional file 7: Figure S7) together with Rnd2 shRNA in embryonic E14.5 cortical cells. Our results show that while treatment with Bacurd2(3A):Rnd2 improved the migration of Rnd 2 shRNA-treated cells, the proportion of GFP-labelled cells within the CP remained significantly decreased compared with control condition (Figure $8 \mathrm{~A}, \mathrm{~B} ; 13.43 \% \pm 2.76 \%$ of cells within the CP of Rnd2shRNA-treated cortices versus $26.38 \% \pm 2.06 \%$ in Rnd2shRNA+ Bacurd2(3A):Rnd2 treated cortices versus $39.37 \% \pm 2.57 \%$ of cells within the CP of control samples; $F_{8,39}=17.36 ; P<0.0001$; two-way ANOVA; post hoc $t$-test $\left.{ }^{* * *} P<0.0001\right)$. 


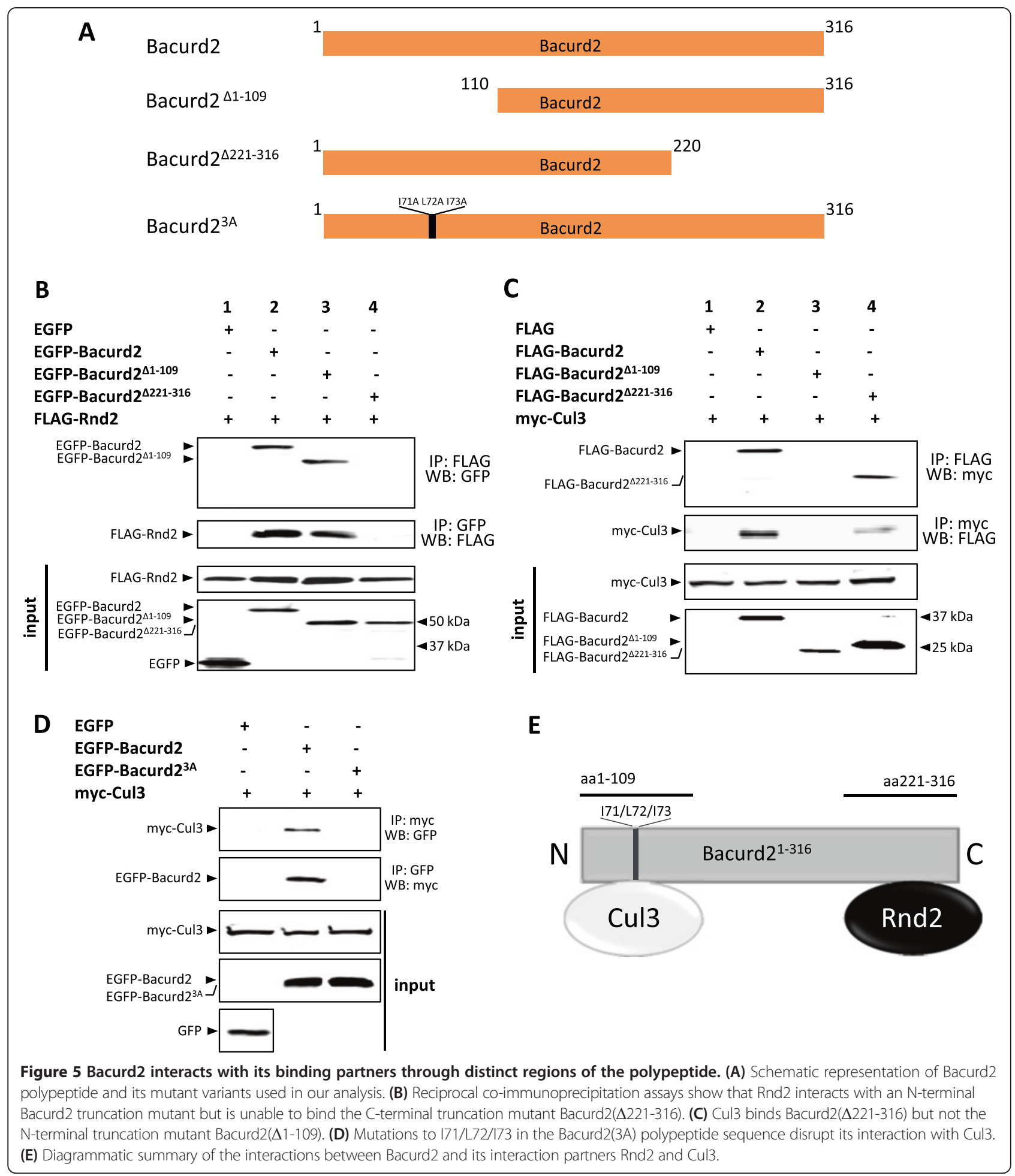

We previously demonstrated that Rnd2 controls the morphological transitions undertaken by migrating neurons as they reach the $\mathrm{CP}$, including their multipolar-to-bipolar transition as they leave the IZ and enter the CP [6,7]. Thus, we analysed the migration index of GFP-labelled cells in our current rescue experiments to understand how neurons enter the IZ (Figure 8C) and the CP (Figure 8D,E). As a control experiment, we first confirmed that Rnd2-deficient cells are defective in their migration from the VZ to the IZ and $\mathrm{CP}$ in a cell autonomous fashion, as previously reported [6,7] (Figure 8C,D,E). We then observed that the IZ migration defect of Rnd2 shRNA-treated cells is restored 


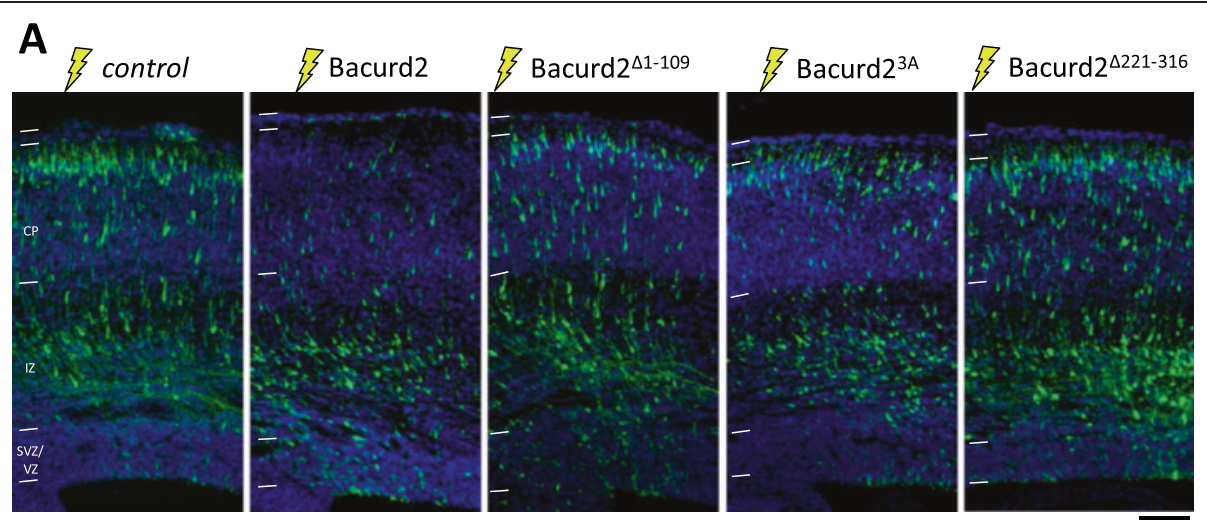

B

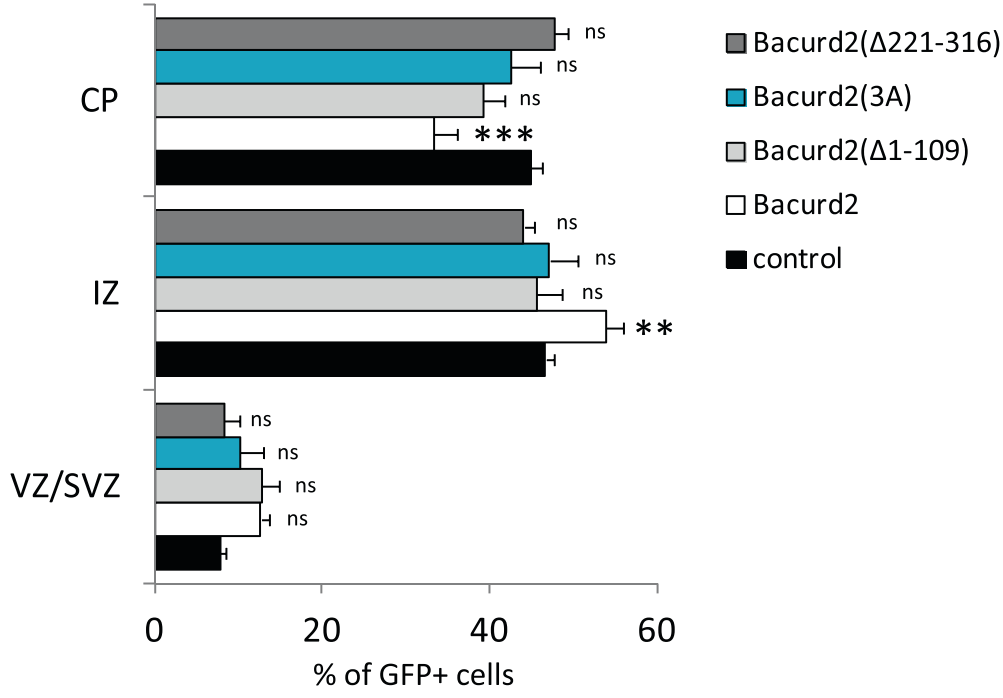

Figure 6 The effect of forced expression of Bacurd2 and its mutated variants on cell migration within the embryonic E17.5 cortex. (A) Coronal sections of E17.5 embryonic cortex following E14.5 in utero electroporation with a bicistronic construct encoding GFP vector only (control), or together with Bacurd2 or its mutant variants. (B) Forced expression of Bacurd2 disrupts the migration of GFP-labelled cells in the embryonic cortex, when compared with control treatment. On the other hand, forced expression of the N-terminal mutants Bacurd2( $\Delta 1-109)$, Bacurd2(3A) or the C-terminal Bacurd2( $\Delta 221-316)$ variant did not significantly disrupt the migration of cells $(N>4,000$ cells counted from four to six brains per condition. Distribution of GFP-labelled cells within the VZ/SVZ, IZ and CP of the E17.5 cortex; $F_{8,96}=5.38$; two-way ANOVA followed by Bonferroni's post hoc test which compares each column to control; ${ }^{*} P<0.05$; ${ }^{* * *} P<0.001$ ). Scale bar, $100 \mu \mathrm{m}$.

with either the Bacurd2:Rnd2 or Bacurd2(3A):Rnd2 to levels which are not significantly different to control profile (Figure $8 \mathrm{C}$ ). In contrast, the defective CP-entry of Rnd2-deficient cells was efficiently restored only when Bacurd2:Rnd2 was co-delivered, but not with Bacurd2 (3A):Rnd2 (Figure 8D). Furthermore, we found that the defective intracortical distribution of Rnd2-deficient cells was only corrected by co-delivery of Bacurd2:Rnd2, but not with Bacurd2(3A):Rnd2 (Figure 8E).

Finally, we analysed GFP-labelled neurons within the IZ and CP to determine whether the abnormal morphologies of Rnd2 shRNA-treated neurons could be corrected by co-delivery of Bacurd2:Rnd2. We first investigated the morphologies of neurons within the IZ of Rnd2 shRNA electroporated brains and found a significant increase in the proportion of multipolar-shaped neurons compared with control treatment, a result which is consistent with our previous reports describing failed multipolar-tobipolar transition of Rnd2-deficient cells $[6-8,10,11]$ (Figure $8 \mathrm{~F}$ ). Also, we observed that co-delivery of Rnd2 restores the morphologies of Rnd2 shRNA-treated neurons to a distribution which is not significantly different to control treatment. In contrast, we found that the morphological profiles of Rnd2 shRNA + Bacurd2:Rnd2 treated cells within the IZ and CP were restored to a profile resembling control condition, as were $R n d 2$ shRNA cells co-treated with Bacurd2(3A):Rnd2 (Figure 8G,H). Taken together, our results collectively demonstrate that Bacurd2 coordinates cell migration within the embryonic cortex and influences the morphological transitions of immature neurons as they transit through the IZ, as well as when their radial distribution within the $\mathrm{CP}$. Despite the 


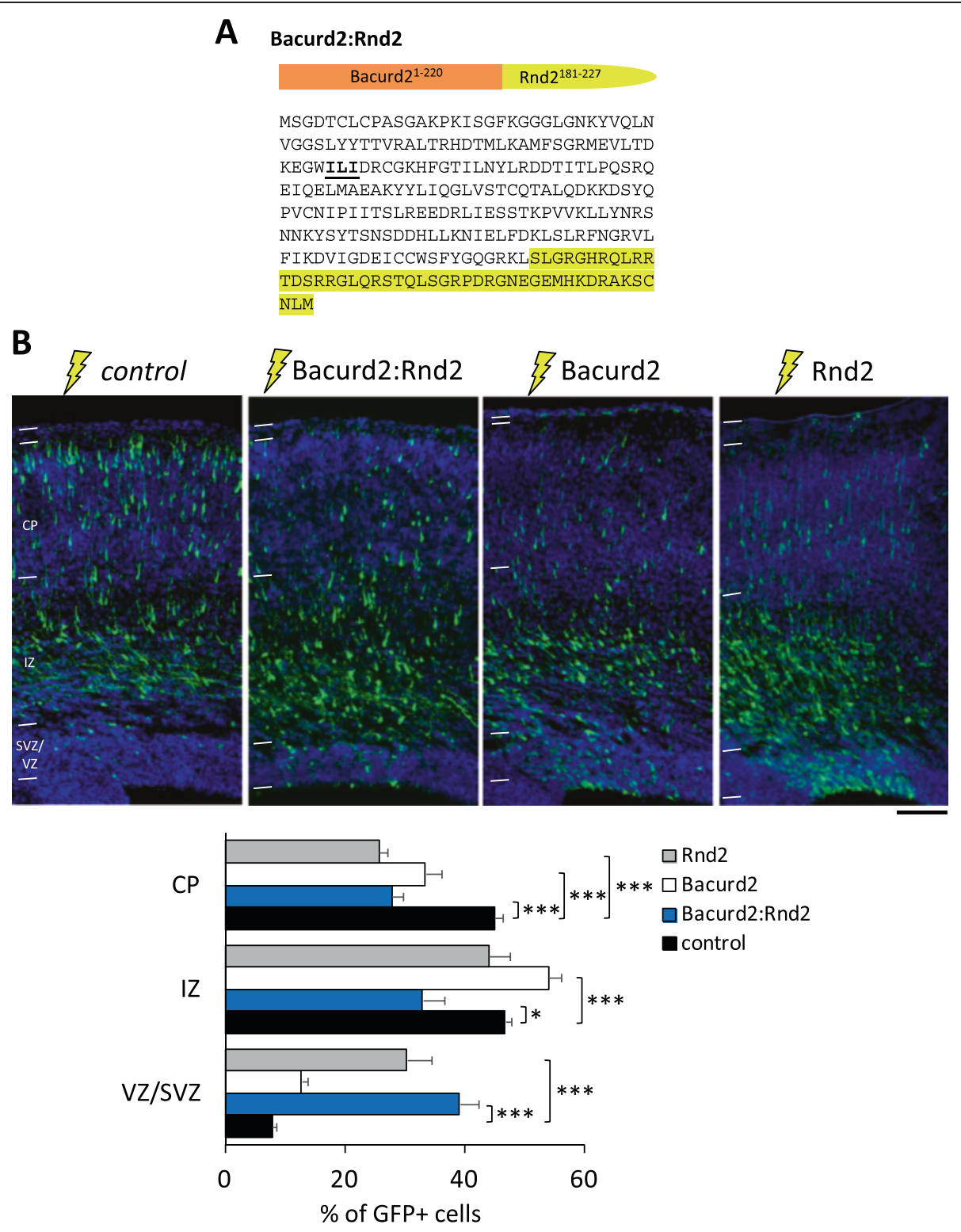

Figure 7 Forced expression of Bacurd2:Rnd2 impairs radial migration in vivo. (A) Illustration of the protein resulting comprising the $\mathrm{N}$-terminal region of Bacurd2(1-220) together with the C-terminal region of Rnd2 which mediates the migration of embryonic cortical neurons in vivo [7]. (B) Forced expression of Bacurd2:Rnd2 impairs cell migration within the embryonic E17.5 cortex, as do cells which overexpress either Bacurd2 or Rnd2 ( $N>2,400$ cells counted from three to four brains per condition; $F_{6,63}=55.34 ; P<0.0001$; two-way ANOVA followed by Bonferroni's post hoc test). Scale bar, $100 \mu \mathrm{m}$.

caveat that our Bacurd2:Rnd2 chimeric construct represents an artificial model of a Bacurd2-Rnd2 signal transducer, our results suggest that Bacurd2 and Rnd2 promote cell migration within the embryonic cortex.

\section{Discussion}

We previously reported that Rnd2 regulates the migration of newborn embryonic cortical neurons [6,7]; hence, we wanted to clarify the downstream signalling pathway through which Rnd2 modulates this activity. In this study, we have identified Bacurd2 as an interacting partner to Rnd2 which influences radial migration during cerebral cortex development. Notably, both Rnd2 and RhoA signalling are crucial to radial migration $[6,7,19]$, and Bacurd2 suppresses RhoA to influence cell migration in vitro [18]. Therefore, we were motivated to characterise the neuronal functions for Bacurd2 within the embryonic cortex. We find that disruptions to Bacurd2 impair cell migration and alter the multipolarto-bipolar transition of embryonic cortical neurons. Thus, our study introduces Bacurd2 as a new player in neuronal development. 


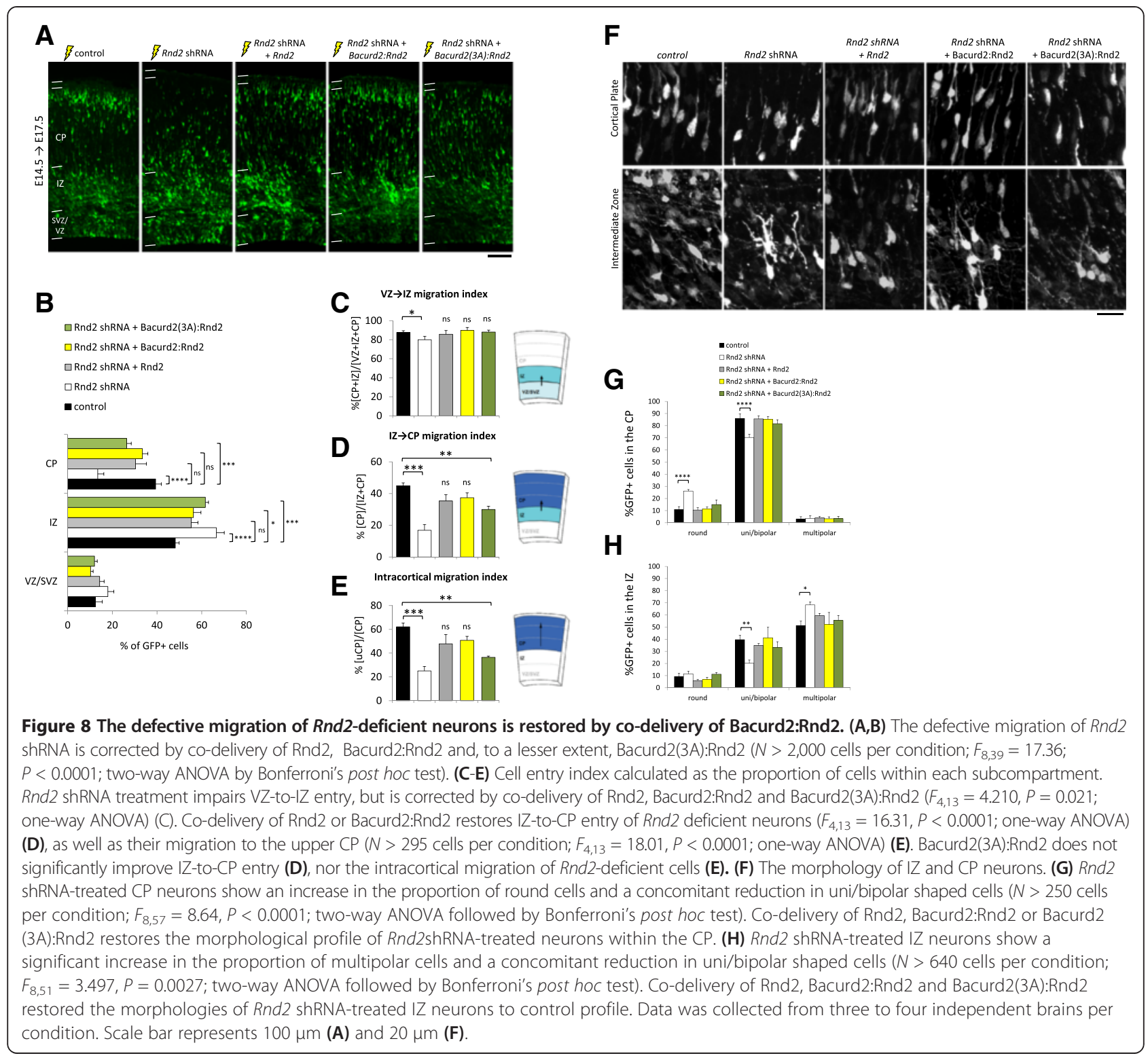

The ability for immature neurons to migrate is a function which is sensitive to Rnd2 levels, with too much or too little disrupting this process $[6,7,10]$. Given the role for Bacurd2 in targeting RhoA for degradation by the Cul3 ubiquitin ligase complex, it is possible that Bacurd2 may act as a substrate adaptor for the degradation of Rnd proteins. As such, Bacurd2 could target Rnd2 for degradation via the Cul3 ubiquitin ligase complex so as to promote radial migration. However, the role for Bacurd2 in radial migration is also likely to be mediated through RhoA regulation as well. In the future, it will be important to determine the relative contributions of both of these postulated signalling mechanisms for Bacurd2 which influence the development of cerebral cortical neurons.
In our functional studies, we found that truncation of the C-terminal region of Bacurd 2 abolishes its binding to Rnd 2 and disrupts its cell migration functions in embryonic cortical cells. Furthermore, a truncation of the $\mathrm{N}$-terminal region of Bacurd2 or the introduction of missense mutations I71A/L72A/I73A within its BTB-domain (both of which disrupts its binding to Cul3) similarly abolishes its effects on cell migration in vivo. From these findings, we surmise that the ability for the Bacurd2 polypeptide to control cell migration relies on its $\mathrm{N}$ - and C-terminal domains. With the knowledge that $\mathrm{Cul} 3$ and Rnd2 interact with the $\mathrm{C}$ - and $\mathrm{N}$ - termini of Bacurd2, respectively, together with our evidence that these proteins are detected throughout the course of brain development, 
our future studies will address their combined roles within the context of neurodifferentiation.

A significant challenge in our study was to address how Bacurd2 and Rnd2 might signal to promote cell migration. We took a drastic approach by tethering a Rnd2(aa181227) polypeptide sequence to the C-terminal (aa221-316) region of Bacurd2. We found that Bacurd2:Rnd2 could restore the defective migration of Rnd2shRNA-treated neurons within the embryonic cortex. But, how can we explain the basis for this rescue? One possible explanation relates to the migration-promoting functions of Rnd2 and Bacurd2. In the case of Rnd2, it was reported that its aa181-227 C-terminal region is important for subcellular localisation and promotion of activity within the perinuclear region of immature neurons to signal migration $[7,20]$. On the other hand, studies with a triple I71A/ L72A/I73A point mutation variant of Bacurd2(3A) protein show an importance for Cul3 binding to signal cell migration in a wound-healing assay [18] as well as embryonic cell migration (this study). When we attempted to reconcile these findings through our own experimentation, our results show that Bacurd2:Rnd2 restores the migration of Rnd2 shRNA-treated neurons. While the roles for both Bacurd 2 and Rnd2 in neuronal development remain to be better understood, it will be important to determine if these molecules are important for the terminal differentiation of cortical neurons, including their dendritic arborisation and synaptic connectivity. More broadly, given the roles for Bacurd and Rnd proteins in the regulation of the early steps of neurogenesis $[7,12,21,22]$, a better understanding of their combined signalling activities will reveal their specific contributions to the development and function of cerebral cortical neurons in health and disease.

\section{Conclusions}

The molecular regulation of radial migration is complex and involves multiple signalling factors which promote directional movement as well as neurite outgrowth as immature neurons position themselves within the developing cerebral cortex. We have identified Bacurd2 as a new player which promotes the migration of immature cortical projection neurons in a concentration-sensitive manner. In addition, we have characterised Bacurd2 as a novel interacting partner to Rnd2, a known regulator of radial migration within the embryonic cortex. Our discovery supports the notion that Rnd2 interacts with specific binding partners (such as Bacurd2) to control neuronal migration during cortical development.

\section{Methods}

Animals - Mice were housed, bred and treated within the animal facilities at Monash University. All animal procedures are approved by the Animal Ethics Committee within Monash University (Licenses MARP/08-104 and MARP/2012/068) and are compliant with guidelines provided by the National Health and Medical Research Council of Australia.

Cloning, plasmids and siRNA - cDNA for murine Bacurd2 (also known as Tnfaip1) was amplified from Invitrogen clone accession number BC003906 using primers, 5'-CATCATCAATTGatgtcaggggacacctgtctg 3' (forward) and 5'-CATCATCAATTGtcagtc acgatgagtggactg-3' (reverse) (Invitrogen, Grand Island, USA). Truncation mutants were constructed by the standard PCR cloning strategy or DNA synthesis of the entire cDNA (Life Technologies, Carlsbad, USA). Complementary DNA (cDNA) fragments were cloned into the EcoRI site of pEGFP-C2 (Clontech, Mountain View, USA) and pCIG2Flag. Expression constructs for Rnd2 and Rnd3 were previously described [2]. The Bacurd2:Rnd2 cDNA encodes the following polypeptide which is engineered as a fusion between aa1-220 of Bacurd2 and aa181-227 of Rnd2: MSGDTCLCPASGAKPKISGFKGGGLGNKYVQLNVGG SLYYTTVRALTRHDTMLKAMFS GRMEVLTDKEGWIL IDRCGKHFGTILNYLRDDTITLPQSRQEIQELMAEAKY YLIQGLVSTCQTALQDKKDSYQPVCNIPIITSLREEDRLI ESSTKPVVKLLYNRSNNKYSYT SNSDDHLL KNIELFDK LSLRFNGRVLFIKDVIGDEICCWSFYGQGRKLSLGRGH RQLRRTDSRR GLQRSTQLSGRPDR GNEGEMHKDRAKS $C N L M$ (the Rnd2 C-terminal polypeptide sequence is represented in italics, while the amino acids in bold are targeted for alanine substitution in the Bacurd2(3A):Rnd2 construct). All constructs were sequenced verified and plasmids produced using PureYield ${ }^{\mathrm{mi}}$ Midi-prep kits (Promega, Madison, USA).

Immuno-precipitation and Western blotting - Six embryonic 14.5 mice brains were homogenised (ProScientific Pro200 homogenizer, ProScientific, Oxford, USA) in icecold lysis buffer (20 mM Tris (pH 7.5), $150 \mathrm{mM} \mathrm{NaCl}, 1 \%$ IGEL-PAL and $0.1 \%$ SDS supplemented with protease inhibitor). The resultant lysate was sonicated then centrifuged at $10,000 \times g$ for $20 \mathrm{~min}$ at $4^{\circ} \mathrm{C}$ before transferring to a fresh tube. The cleared lysate was then incubated with antibodies: mouse IgG (Millipore, Billerica, USA) and mouse anti Bacurd2 (Abmart, Shanghai, China) overnight at $4^{\circ} \mathrm{C}$ with rotation. The solution was then incubated with protein A sepharose beads for $2 \mathrm{~h}$ at $4^{\circ} \mathrm{C}$ and then washed three times with the cold protein lysis buffer. Immunoprecipitated proteins were eluted with loading buffer containing $50 \mathrm{mM}$ DTT and samples were analysed on a $10 \%$ SDS page gel. Following membrane transfer, the membranes were incubated with anti-Rnd2 antibodies, followed by secondary goat-anti-rabbit antibody (Li-Cor IRDye 680 LT). Signals were detected with Odyssey infrared imaging system (Li-Cor 9201-02, Lincoln USA) for analysis. Native antibodies to Bacurd2 (epitope is SPSEDEDTFE Abmart) and Rnd2 (sc-33543; Santa Cruz Biotechnologies, Santa Cruz, USA) were used. 
In utero electroporation - In utero electroporation experiments are performed as described [23]. High-quality, low endotoxin plasmid preparations (Qiagen, Limburg, The Netherlands) of DNA vectors were injected at $1 \mu \mathrm{g} / \mu \mathrm{l}$ for each plasmid, together with Fast Green $(0.05 \%$, Sigma, St. Louis, USA). For RNAi experiments, Dharmacon siRNA targeting pools for Bacurd2 were injected at $10 \mu \mathrm{M}$ concentration together with GFP expression plasmid at 1 $\mu \mathrm{g} / \mu \mathrm{l}$ concentration. Following recovery from in utero electroporation, the mice were sacrificed by cervical dislocation, and the embryonic brains were harvested by dissection in cold PBS and preserved for tissue processing, cryosectioning $(16 \mu \mathrm{m})$ and fluorescence immunostaining. Images of brain sections were captured on an epifluorescence microscope (Olympus, Tokyo, Japan) equipped with a CCD camera (SPOT, Sterling Heights, USA). Subdivisions of the embryonic cortex (VZ/SVZ, IZ and CP) were identified based on cell density as visualised with 4'6-diamidino-2-phenylindole (DAPI) staining. Cell counting was performed blind to the condition on representative fields of sections of electroporated brains using ImageJ software.

\section{Additional files}

Additional file 1: Figure S1. Identification of Bacurd2 as a binding partner to Rnd2 in a yeast two-hybrid assay. Representation of results of a yeast two-hybrid interaction screen using an Rnd2 bait which lacks its C-terminal (CAAX) motif. (A) Identification of multiple CDNA prey encoding Bacurd2 polypeptide. Complementation assays confirm association of Bacurd2 preys with Rnd2 bait. (B) Growth of yeast transfected with Rnd2 bait and Bacurd2 prey construct on nutritional selection medium (lacking histidine and adenine). Double-transfected yeast harbouring Bacurd2 prey and pLaminC bait, or Bacurd2 prey with $\mathrm{p} 53$ bait do not grow on this selection medium.

Additional file 2: Figure S2. Immunodetection of Bacurd2 and Rnd2. (A) Western blotting of HEK293T cell lysates transfected with FLAG-Bacurd2 construct and immunoblotted with our mouse monoclonal antibody. A specific signal of approximately $37 \mathrm{kDa}$ is detected, which corresponds to the FLAG-Bacurd2 signal in an immunoblot with FLAG antibody. (B) Western blotting of HEK293T cell lysates transfected with FLAG-Rnd2 construct and immunoblotted with a rabbit polyclonal antibody (Santa Cruz Biotechnologies, Santa Cruz, USA). A specific signal of approximately $25 \mathrm{kDa}$ is detected and which corresponds to the FLAG-Rnd2 signal in an immunoblot with FLAG antibody. (C) Western blotting using antibodies to Bacurd2, Rnd2 and Cul3 on mouse brain lysates harvested from the indicated timepoints during mouse brain development. The signal for $\beta$-actin was used as a loading control. (D-E) Fluorescence immunostaining of E14.5 embryonic cortex using preimmune serum (D) or Bacurd2 antibody ( $\mathrm{E}$, green signal). Nuclei were counterstained with DAPI. Scale bar, $50 \mu \mathrm{m}$.

Additional file 3: Figure S3. Perturbations to Bacurd2 do not disturb neuronal differentiation. (A) GFP-labelled cells which co-express Tuj1 (arrow) or do not co-express Tuj1 (arrowheads) are identified in a representative section of E17.5 embryonic cortex. (B) Quantification studies of the proportion of GFP-labelled cells which co-express the neuronal marker Tuj1 ( $N>1,700$ cells counted from at least three brains per condition; one-way ANOVA followed by Bonferroni's post hoc $t$-test) reveal no significant difference in GFP+/Tuj $1+$ cells following overexpression or knockdown of Bacurd2 $\left(F_{2,13}=1.6 ; P=0.24 ; P>0.5\right)$. (C) Two-way ANOVA analysis of GFP+/Tuj1 - cells in each cortical subcompartment reveals no significant difference in their distribution following overexpression or knockdown of Bacurd2 $\left(F_{4,39}=0.44 ; P=0.77 ; P>0.5\right)$. Graph plots mean \pm SEM. Scale bar, $50 \mu \mathrm{m}$.
Additional file 4: Figure S4. The defective migration of Bacurd2 siRNA-treated cells is augmented by co-delivery of human BACURD2. (A) To ask if co-treatment with $0.4 \mu \mathrm{g} / \mu \mathrm{l}$ or $1.0 \mu \mathrm{g} / \mu \mathrm{l}$ of BACURD2 construct restores their migration of siRNA-treated cells, we performed two-way ANOVA followed by Bonferroni's multiple comparisons test. We found that treatment with either concentrations of BACURD2 improved the migration of cortical cells ( $N>1,450$ cells counted per condition, $\left.F_{6,45}=15, P<0.0001\right)$. (B) An analysis of their intracortical distribution (that is within the lower, medial and upper (P) reveals that the defective migration of Bacurd2 siRNA-treated cells is restored with co-delivery of $0.4 \mu \mathrm{g} / \mu \mathrm{l}$ of BACURD2 construct, but treatment with $1.0 \mu \mathrm{g} / \mu \mathrm{l}$ of BACURD2 did not restore their positioning within the upper $\mathrm{CP}(N>500$ cells per condition, $F_{6,42}=15.27, P<0.0001$; two-way ANOVA followed by Bonferroni's post hoc test). Graph plots mean \pm SEM. Scale bar represents $100 \mu \mathrm{m}$.

Additional file 5: Figure S5. Bacurd2 controls cell shape in vivo. (A) The morphology of neurons within the CP and the IZ in representative brain sections electroporated with control (GFP only) vector, Bacurd2 siRNAs or Bacurd2 siRNAs with the indicated concentrations of BACURD2 expression vector. Arrowheads point to round-shaped cells. (B) Within the $C P$, knockdown of Bacurd2 leads to a significant increase in the proportion of round cells and a decrease in uni/bipolar-shaped cells $\left(N>300\right.$ cells counted from three brains per condition; $F_{6,60}=24.60$; $P<0.0001$; two-way ANOVA followed by Bonferroni's post hoc test; $\left.{ }^{*} P<0.05,{ }^{* * *} P<0.001\right)$. These effects on cell shape are corrected with co-delivery of $0.4 \mu \mathrm{g} / \mu \mathrm{l}$ of BACURD2 construct. (C) Within the IZ, treatment with Bacurd2 siRNAs leads to a significant decrease in the proportion of uni/bipolar-shaped cells and multipolar-shaped cells, with no significant difference in the proportion of round-shaped cells when compared with control. ( $N>500$ cells counted from three brains per condition; $F_{6,48}=$ 22.52; $P<0.0001$; two-way ANOVA followed by Bonferroni's post hoc test; $\left.{ }^{*} P<0.05,{ }^{* * *} P<0.001\right)$. Co-delivery of either $0.4 \mu \mathrm{g} / \mu \mathrm{l}$ of BACURD2 construct or $1.0 \mu \mathrm{g} / \mu \mathrm{l}$ of BACURD2 construct corrects these Bacurd2 siRNA-induced changes to cell shape within the IZ. Scale bar represents $20 \mu \mathrm{m}$.

Additional file 6: Figure S6. Cell shape profiles following treatment with Bacurd2:Rnd2, Bacurd2 or Rnd2. Analysis of the morphologies of IZ and CP neurons following forced expression of Bacurd2:Rnd2, Bacurd2 or Rnd2. There was an interaction between treatment and distribution of cell shapes in the CP $(N>190$ cells from three to four brains per condition; $F_{6,60}=8.332, P<0.0001$; two-way ANOVA followed by Bonferroni's post hoc test) (A) and cells within the $I Z$ ( $N>390$ cells from three to four brains per condition; $F_{6,51}=7.537, P<0.0001$; two-way ANOVA followed by Bonferroni's post hoc test) (B). Graph plots mean \pm SEM.

Additional file 7: Figure S7. Point mutations 171A/L72A/173A alter binding to Cul3. (A), Diagrammatic representation of Bacurd2 full-length polypeptide sequence, along with Bacurd2:Rnd2 contruct, and a Bacurd2 (3A):Rnd2. Yellow indicates the sequence of Rnd2 polypeptide fused to the C-terminal fragment of Bacurd2 $2^{1-220}$ polypeptide (see the 'Methods' section for polypeptide sequence). Bold letters identify substitution mutations I71A/L72A/I73A within the BTB-domain of the Bacurd2 polypeptide fragment which mediates Cul3 binding. (B) Reciprocal co-imunoprecipitation experiments were performed to confirm that the Bacurd2:Rnd2 interacts with Cul3, but not with Bacurd2(3A):Rnd2. Input lanes confirm the presence of all proteins evaluated in this experiment.

\section{Abbreviations}

bHLH: basic helix-loop-helix; CP: cortical plate; E: embryonic day; EGFP: enhanced green fluorescent protein; IZ: intermediate zone; ICP: lower cortical plate; mCP: medial cortical plate; siRNA: small inhibitory RNA; UCP: upper cortical plate; VZ: ventricular zone.

\section{Competing interests}

The authors declare that they have no competing interests.

\section{Authors' contributions}

$\mathrm{JH}$ conceived the study and performed $\mathrm{Y} 2 \mathrm{H}$ experiments with JMD and ZDQ. Biochemical assays, cell culture experiments and protein-interaction assays were performed by SSL, IEG-N and ZDQ, while mouse electroporation 
experiments were performed by ZDQ, IEG-N, JIH, MAH and LN. JS provided critical reagents to the manuscript and advised on experiments. JIH wrote the manuscript with IEG-N, ZDQ and SSL, and all authors commented on the manuscript. All authors read and approved the final manuscript.

\section{Acknowledgements}

This work was supported by a grant from the Australian Research Council (DP130103328) as well as funding from the Victorian Government through the Operational Infrastructure Scheme. The Australian Regenerative Medicine Institute is supported by grants from the State Government of Victoria and the Australian Government. JIH is a recipient of a Career Development Fellowship (NHMRC Australia, ID:1011505), a CJ Martin Training Fellowship (ID:310616) and a project grant 566835 (NHMRC, Australia). This work was also supported by NIH grant R01GM082940 to JDS. We thank Drs Laurent Nguyen and Emilie Pacary and members of the Heng lab for critical reading of the manuscript.

\section{Author details}

${ }^{1}$ EMBL Australia, The Australian Regenerative Medicine Institute, Monash University, Clayton, Victoria 3800, Australia. ${ }^{2}$ Department of Biology, Portland State University, Portland, Oregon 96207, USA. ${ }^{3}$ The Harry Perkins Institute of Medical Research, Perth, Australia. ${ }^{4}$ Centre for Medical Research, The University of Western Australia, Perth, Australia. ${ }^{5}$ Present address: The Harry Perkins Institute of Medical Research, Perth, Australia.

Received: 24 October 2014 Accepted: 23 February 2015

Published online: 31 March 2015

\section{References}

1. Gupta A, Tsai LH, Wynshaw-Boris A. Life is a journey: a genetic look at neocortical development. Nat Rev Genet. 2002;3(5):342-55.

2. Marin $\mathrm{O}$, Rubenstein JL. A long, remarkable journey: tangential migration in the telencephalon. Nat Rev Neurosci. 2001;2(11):780-90.

3. Marin O, Rubenstein JL. Cell migration in the forebrain. Annu Rev Neurosci. 2003;26:441-83. doi:10.1146/annurev.neuro.26.041002.131058041002.131058.

4. Heng Jl, Chariot A, Nguyen L. Molecular layers underlying cytoskeletal remodelling during cortical development. Trends Neurosci. 2010;33(1):38-47. doi:10.1016/j.tins.2009.09.003.

5. Leventer RJ, Guerrini R, Dobyns WB. Malformations of cortical development and epilepsy. Dialogues Clin Neurosci. 2008;10(1):47-62.

6. Heng II, Nguyen L, Castro DS, Zimmer C, Wildner H, Armant O, et al. Neurogenin 2 controls cortical neuron migration through regulation of Rnd2. Nature. 2008:455(7209):114-8. doi:10.1038/nature07198.

7. Pacary E, Heng J, Azzarelli R, Riou P, Castro D, Lebel-Potter M, et al. Proneural transcription factors regulate different steps of cortical neuron migration through Rnd-mediated inhibition of RhoA signaling. Neuron. 2011;69(6):1069-84. doi:S0896-6273(11)00116-4 10.1016/j.neuron.2011.02.018.

8. Heng J, Guillemot F. Proneural proteins and the development of the cerebral cortex. In: Kageyama R, editor. Cortical Development: Neural Diversity and Neocortical Organization. Springer Global; 2013. p. In press.

9. Alfano C, Viola L, Heng Jl, Pirozzi M, Clarkson M, Flore G, et al. COUP-TFI promotes radial migration and proper morphology of callosal projection neurons by repressing Rnd2 expression. Development. 2011;138(21):4685-97. doi:dev.068031 10.1242/dev.068031.

10. Heng Jl, Qu Z, Ohtaka-Maruyama C, Okado H, Kasai M, Castro D et al. The zinc finger transcription factor RP58 negatively regulates Rnd2 for the control of neuronal migration during cortical development. Cereb Cortex. 2013. doi:bht277 10.1093/cercor/bht277

11. Ohtaka-Maruyama C, Hirai S, Miwa A, Heng Jl, Shitara H, Ishii R, et al. RP58 regulates the multipolar-bipolar transition of newborn neurons in the developing cerebral cortex. Cell Rep. 2013;3(2):458-71. doi:S22111247(13)00019-3 10.1016/j.celrep.2013.01.012.

12. Pacary E, Azzarelli R, Guillemot F. Rnd3 coordinates early steps of cortical neurogenesis through actin-dependent and -independent mechanisms. Nat Commun. 2013:4:1635. doi:ncomms2614 10.1038/ncomms2614.

13. Chardin P. Function and regulation of Rnd proteins. Nat Rev Mol Cell Biol. 2006;7(1):54-62. doi:nrm1788 10.1038/nrm1788.

14. Riou P, Villalonga P, Ridley AJ. Rnd proteins: multifunctional regulators of the cytoskeleton and cell cycle progression. Bioessays. 2010;32(11):986-92. doi:10.1002/bies.201000060
15. Heng Jl, Tan SS. Cloning and characterization of GRIPE, a novel interacting partner of the transcription factor E12 in developing mouse forebrain. J Biol Chem. 2002;277(45):43152-9. doi:10.1074/jbc.M204858200 M204858200.

16. LoTurco JJ, Bai J. The multipolar stage and disruptions in neuronal migration. Trends Neurosci. 2006;29(7):407-13. doi:S0166-2236(06)00094-4 10.1016/j.tins.2006.05.006.

17. Noctor SC, Martinez-Cerdeno V, Ivic L, Kriegstein AR. Cortical neurons arise in symmetric and asymmetric division zones and migrate through specific phases. Nat Neurosci. 2004;7(2):136-44. doi:10.1038/nn1172 nn1172.

18. Chen Y, Yang Z, Meng M, Zhao Y, Dong N, Yan H, et al. Cullin mediates degradation of RhoA through evolutionarily conserved BTB adaptors to control actin cytoskeleton structure and cell movement. Mol Cell. 2009;6:841-55. doi:S1097-2765(09)00632-7 10.1016/j.molcel.2009.09.004

19. Nguyen L, Besson A, Heng Jl, Schuurmans C, Teboul L, Parras C, et al. p27kip1 independently promotes neuronal differentiation and migration in the cerebral cortex. Genes Dev. 2006;20(11):1511-24. doi:gad.377106 10.1101/gad.377106.

20. Riou P, Kjaer S, Garg R, Purkiss A, George R, Cain RJ, et al. 14-3-3 proteins interact with a hybrid prenyl-phosphorylation motif to inhibit $G$ proteins. Cell. 2013;153(3):640-53. doi: 10.1016/j.cell.2013.03.044.

21. Azzarelli R, Pacary E, Garg R, Garcez P, van den Berg D, Riou P, et al. An antagonistic interaction between PlexinB2 and Rnd3 controls RhoA activity and cortical neuron migration. Nat Commun. 2014;5:3405. doi:10.1038/ncomms4405.

22. Golzio C, Willer J, Talkowski ME, Oh EC, Taniguchi Y, Jacquemont S, et al. KCTD13 is a major driver of mirrored neuroanatomical phenotypes of the 16p11.2 copy number variant. Nature. 2012;485(7398):363-7. doi:nature11091 10.1038/nature11091.

23. Breuss M, Heng Jl, Poirier K, Tian G, Jaglin XH, Qu Z, et al. Mutations in the beta-tubulin gene TUBB5 cause microcephaly with structural brain abnormalities. Cell Rep. 2012;62(6):1554. doi:S2211-1247(12)00414-7 10.1016/ j.celrep.2012.11.017

\section{Submit your next manuscript to BioMed Central and take full advantage of:}

- Convenient online submission

- Thorough peer review

- No space constraints or color figure charges

- Immediate publication on acceptance

- Inclusion in PubMed, CAS, Scopus and Google Scholar

- Research which is freely available for redistribution 